\title{
Experimental investigation of the check valve behaviour when the flow is reversing
}

\author{
D. Himr ${ }^{\mathrm{a}}$, V. Habán, M. Hudec, and V. Pavlík
}

Brno University of Technology, Faculty of Mechanical Engineering, Energy Institute, Victor Kaplan Department of Fluid Engineering, Technická 2, 616 69, Brno, Czech Republic

\begin{abstract}
Check valve in a pipeline is supposed to prevent the reverse flow and to allow the flow in the positive direction. The construction of check valves follows these requirements, but the check valve must not cause pressure pulsations in transients. It means when the fluid is accelerating or decelerating. The article describes an experimental investigation of a swing check valve when the flow is changing its direction. The check valve was placed in an experimental circuit, where the pressure on the upstream and downstream side of the valve was measured and the current value of flow rate was determined. The goal was to simulate conditions in the real system, where the check valve slam had been observed.
\end{abstract}

\section{Introduction}

The check valve is an important part of hydraulic systems and allows flow in one direction and prevent the reverse flow through the pump. The check valve can be also installed in the system to limit the pressure surge induced by the pump failure [1]. The basic requirements on the check valve are:

- the low pressure loss in the positive direction,

- no flow in the opposite direction, it means good sealing when the valve is closed.

Producers, usually, give static characteristics of check valves such as a pressure drop dependence and opening dependence on the flow rate, the cracking pressure (the pressure when the check valve starts opening) and the minimal flow rate when the check valve is fully open.

The producer also can say whether the valve is appropriate for the pulsating flow, sludge water, horizontal or vertical pipe and so on. These parameters together with the reliability and maintenance difficulty allow choosing the right check valve to reduce operational costs.

But, there is a problem with predicting the dynamic behaviour of the check valve in the particular system. It is, maybe, more important than properties written above. When the fluid flow changes its orientation, the check valve is desired to close before the back flow velocity becomes too high otherwise the check valve disc slams and makes the high pressure surge, which is often connected with the column separation particularly on the upstream side of the check valve. The lower static pressure in the pipeline the greater column separation probability. The slam can lead to severe damages of the check valve and/or the whole system [2], [3], [4].

The check valve also can make problems with selfexcited pressure pulsations: The oscillating control valve at the downstream end of pipe causes troubles with check

\footnotetext{
a e-mail: himr@fme.vutbr.cz
}

valve slam at the upstream end [5]. An example of the pressure surge due to the check valve slam after the pump stoppage is shown in the figures 1 and 2. The slam also causes strong mechanical vibrations of pipeline with acceleration about $20 \mathrm{G}$ in this case [4].

McElhaney made an extensive analysis of check valve failures in the nuclear industry, because these failures are well described, and they focused on correlation between the valve design and failure mode and failure distribution [6].

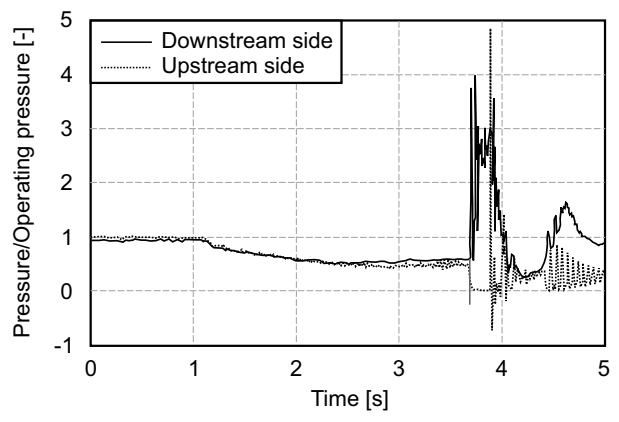

Fig. 1. Pressure surge at the check valve due to slam [4]

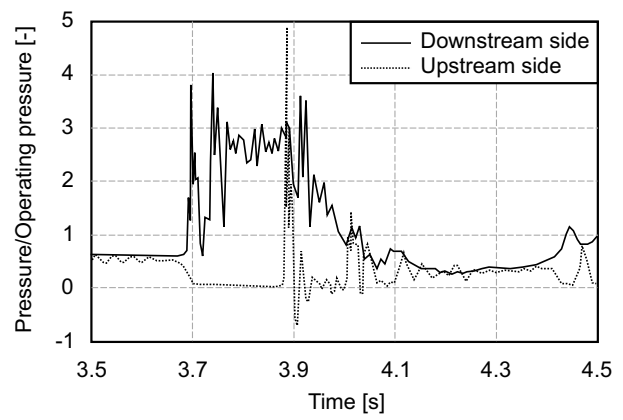

Fig. 2. Pressure surge at the check valve due to slam - detail [4] 
In severe pumping applications, almost all basic check valves will slam, and in extremely mild applications, hardly any check valves will slam [7]. But it is difficult to predict probability of the check valve slam between these two extremes.

The calculation of the system deceleration is important to say whether the check valve will slam or not. The deceleration is calculated from the original forward flow velocity and time period when the velocity reaches zero. It depends on many factors: complexity of the system, friction, pump inertia, static head and so on. Thus, the deceleration can be hardly determined without direct measurement or at least a numerical simulation of the flow in the pipe line system.

The maximal velocity of the reverse flow depends on the check valve construction and on the system deceleration. The higher deceleration the higher reverse velocity can develop before the check valve closes. Then, the pressure surge follows the Joukowski equation.

The reverse velocity dependence on the flow deceleration according to [8] is shown in the figure 3 and a dependence published in [9] is plotted in the figure 4 .

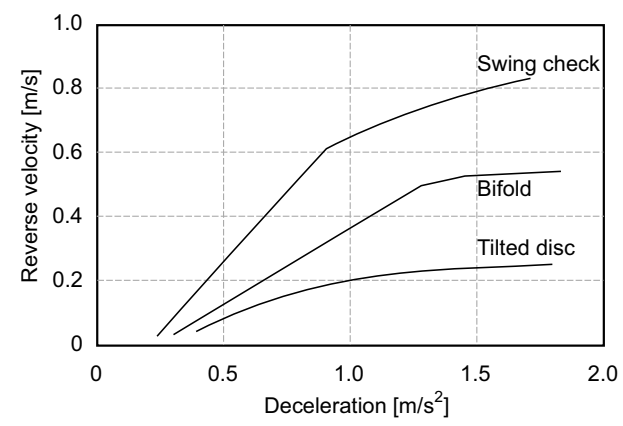

Fig. 3. Reversal velocity for different constructions of the check valve by [8]

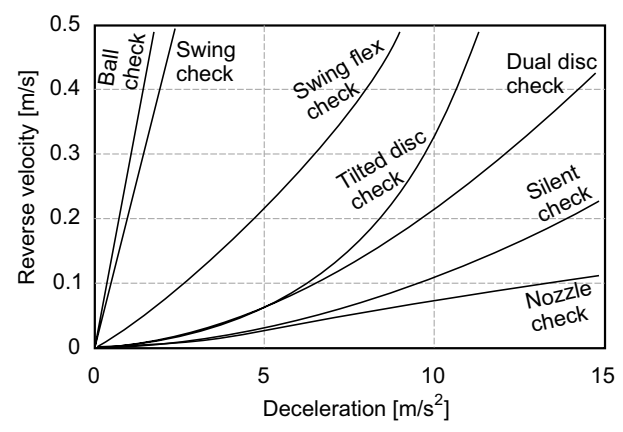

Fig. 4. Reversal velocity for different constructions of the check valve by [9]

One can see that results (e. g. of the tilted disc check valve) are quite different so it is obvious that the reversal velocity does not depends only on the deceleration and type of the check valve, but, probably, also on the specific construction, size, mass, placement,... The system deceleration gives just a basic hint whether the check valve tends to slam or not.
Table 1. Stand specifications

\begin{tabular}{lll}
\hline Entry & Value & Unit \\
\hline Pipe diameter & 0.1 & $\mathrm{~m}$ \\
Maximal flow rate & 31 & $1 \mathrm{~s} \mathrm{~s}^{-1}$ \\
Maximal flow velocity & 3.95 & $\mathrm{~m} \mathrm{~s}^{-1}$ \\
Length of discharge pipe & 17.3 & $\mathrm{~m}$ \\
Static head & 7.5 & $\mathrm{~m}$ \\
Volume of bottom tank & 1 & $\mathrm{~m}^{3}$ \\
Volume of top tank & 0.8 & $\mathrm{~m}^{3}$ \\
Pipe material & steel & \\
Maximal pump speed & 1450 & $\mathrm{rpm}$ \\
\hline
\end{tabular}

\section{Experiment}

An experimental stand has been built and serves for the examination of the check valve behaviour, when the flow orientation is changing. The experiment is a part of cooperation with MSA company, a producer of valves, and the goal is to design a check valve, which does not slam in extreme operating conditions. MSA company provided a swing check valve DN 100, which tends to slam and it is the first check valve of a row, which is going to be tested.

The stand consists of the pump, which collects water from the bottom tank. The tested check valve is placed in the horizontal part of the discharge pipe $2.3 \mathrm{~m}$ above the water level in the bottom tank. The discharge pipe ends in the top tank. The water from top tank returns back through the returning pipe and the overspill pipe (see figure 5 and table 1).

Pressure sensors (range $0-1 \mathrm{MPa}$, uncertainty $0.25 \%$ of the range, sampling frequency $1 \mathrm{kHz}$ ) are placed $0.3 \mathrm{~m}$, $1.4 \mathrm{~m}$ and $2.5 \mathrm{~m}$ before the check valve and $0.41 \mathrm{~m}, 1.4 \mathrm{~m}$ and $2.38 \mathrm{~m}$ behind the check valve in the steady flow direction. The flow rate is measured with the electromagnetic flowmeter $(0-80 \mathrm{l} / \mathrm{s}$, uncertainty $0.5 \%$ of measured value). One accelerometer $(-50 \mathrm{G}-50 \mathrm{G}$, uncertainty $1 \%$ of measured value, sampling frequency $1 \mathrm{kHz})$ is placed on the check valve body and another one is placed on the pump. Accelerometers should help to identify an exact time of some events after pump disconnection. The pump speed is measured with the laser and one mark on the motor shaft.

The pump speed is controlled with a frequency converter to control the flow rate and flow deceleration. Unfortunately, there was a problem with the converter during the experiments and it was not possible to control the deceleration rate.

The check valve design does not allow measuring position of the disc, but check valves, which are going to follow, have windows to see the exact position of the disc and allow PIV measurement of the flow field in the check valve body.

Three pressure sensors on both sides of the valve allow calculating the wave speed and flow rate during deceleration by time-pressure method. Using the flowmeter is not possible due to its great integration constant so the flowmeter can measure just the steady flow rate.

The experiment procedure is following:

1. Start of pump and run with the specific speed to have a desired flow rate (from $10 \mathrm{l} / \mathrm{s}$ to $30 \mathrm{l} / \mathrm{s}$ ). It was not possible to keep the flow for time longer than $30 \mathrm{~s}$, because the overspill aerated water. The air decreases the wave 


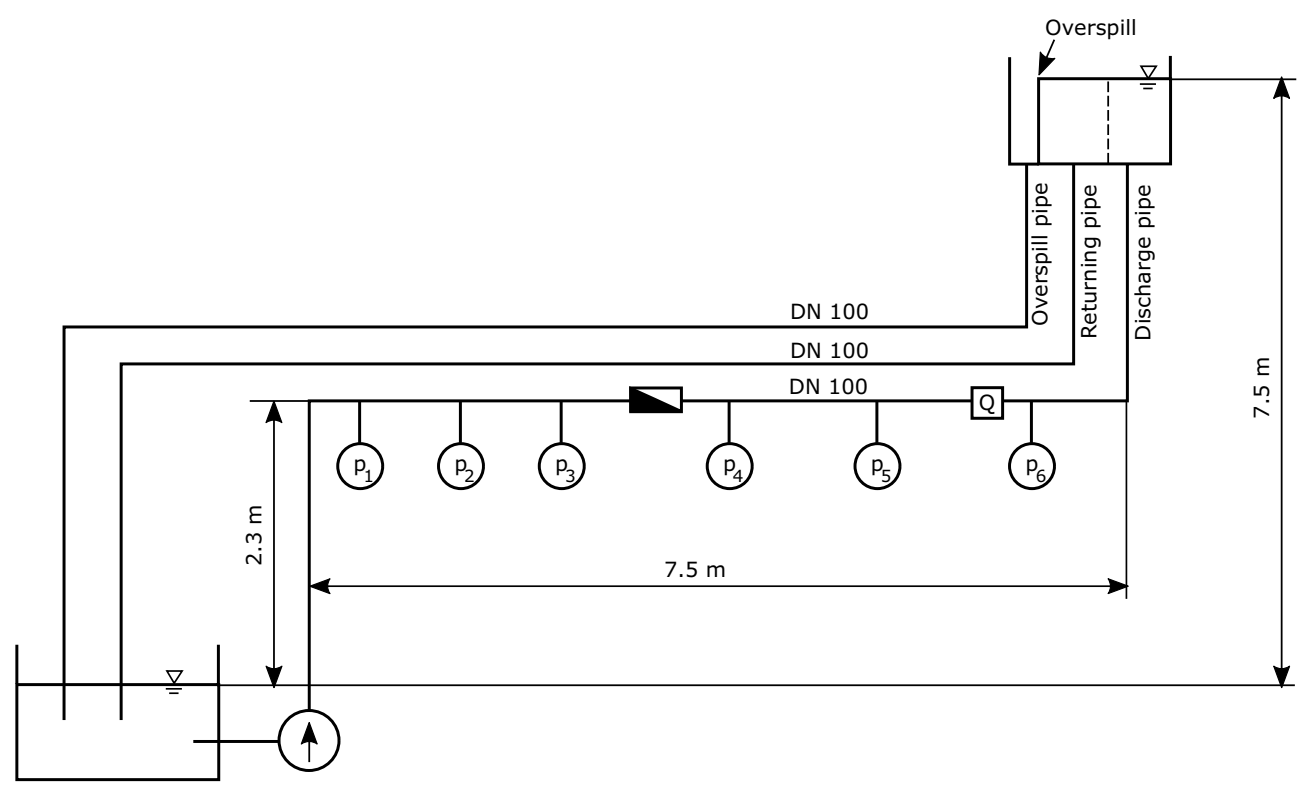

Fig. 5. Experimental stand

speed, which directly influences the height of pressure peaks according to Joukowski equation, and changes the system response [10], [11].

2. As soon as the steady flow was reached the pump was disconnected. Since the pipe line is quite short, it was possible to turn off the pump after five seconds after the start.

3. The measurement was recorded from the start of pump until all transients after the pump stop were finished. Then, it is possible to evaluate: cracking pressure, flow deceleration, maximal back flow velocity, pressure surge in front of and behind the check valve, wave speed and acceleration connected with mechanical vibration on the check valve body.

\section{Results}

The pump was stopped from the four different steady flow rates: $10 \mathrm{l} / \mathrm{s}, 15 \mathrm{l} / \mathrm{s}, 20 \mathrm{l} / \mathrm{s}, 27 \mathrm{l} / \mathrm{s}$ and $30 \mathrm{l} / \mathrm{s}$. An example of results for stop from $15 \mathrm{l} / \mathrm{s}$ is shown in the figures 6 to 12 .

The instant pump speed is evaluated from scanning the spot on the motor shaft. It allows identifying the time of pump disconnection from the grid and the time, when the shaft definitely stopped. These points are, respectively, labelled with numbers 1 and 6 in the figures.

Since there was only one mark on the rotor shaft, there is not problem to count number of revolutions and calculate the exact speed (there is not any demand on the exact position of the mark), but (because we are talking about an unsteady rotation) the slower shaft rotates the less accurate evaluated speed is. That is the reason why the last evaluated speed of the shaft is $85 \mathrm{rpm}$. The last mark was read at the time $3.2 \mathrm{~s}$, the shaft stopped after that.

Pressure upstream of the check valve (figure 7) exhibits the pump disconnection (point 1), when the pressure started decreasing. Time, when the flow changes orientation and starts flowing backward, is marked with point 2 . This very weak peak can be easily overlooked.

Point 3 labels time instant, when the check valve starts closing and point 4 is the moment, when the disc hits the

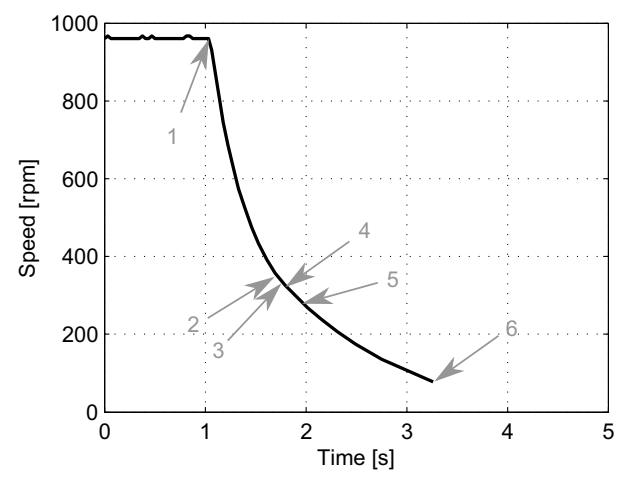

Fig. 6. Pump deceleration from steady flow rate $151 / \mathrm{s}$

seat. (The column separation occurred here in cases with greater initial flow rate).

Then, the highest peak emerges and pressure pulsations are damped, but the pump shaft still rotates. The shaft stop (point 6) caused amplification of the following pulsations about $100 \%$, but the absolute value was still low, thus it did not cause any significant event. These amplified pulsations were damped within 1 second.

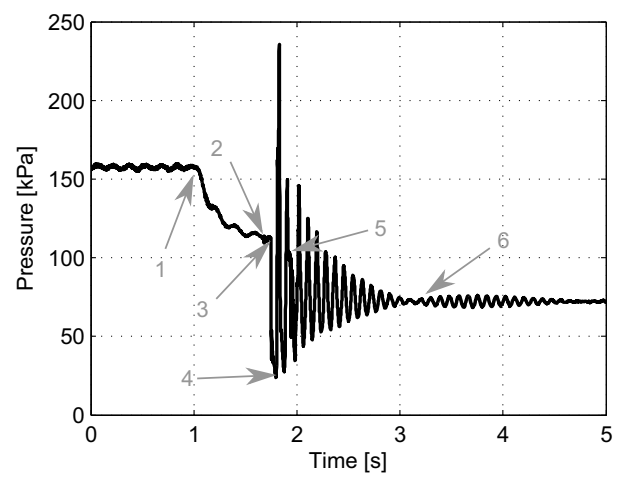

Fig. 7. Pressure in front of the check valve during the transient 
The pressure downstream of the check valve (figure 8) allows identifying the time when the pump was disconnected (point 1). Points 3 and 4 mark moment when the disc started and finished closing.

The following pressure drop goes to the value, which is lower than one would expect (point 5). The same point in the figure 7 shows that the pressure peak is lower than it should be, when the previous and subsequent peaks are considered. This is the time instant when the disc reopens (pressure at the upstream side of the disc is greater than at the downstream side) and closes again. The figure 9

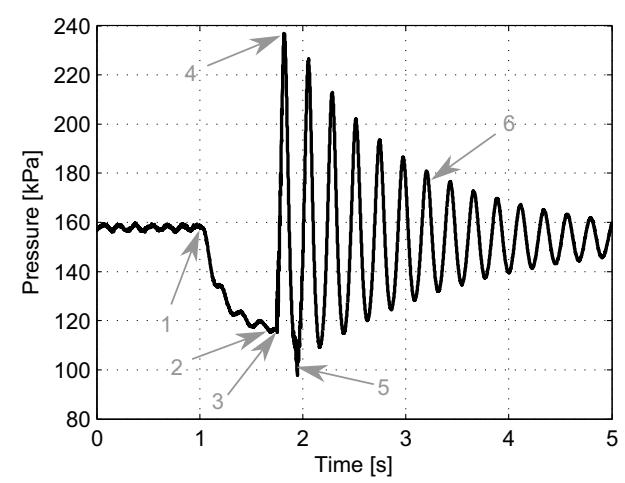

Fig. 8. Pressure behind the check valve during the transient

shows the pressure difference on the check valve. A positive value corresponds to the situation, when the pressure downstream of the valve is greater than upstream of the valve, so the check valve is closed. Negative value at the point 5 supports the statement that the disc reopens at that moment.

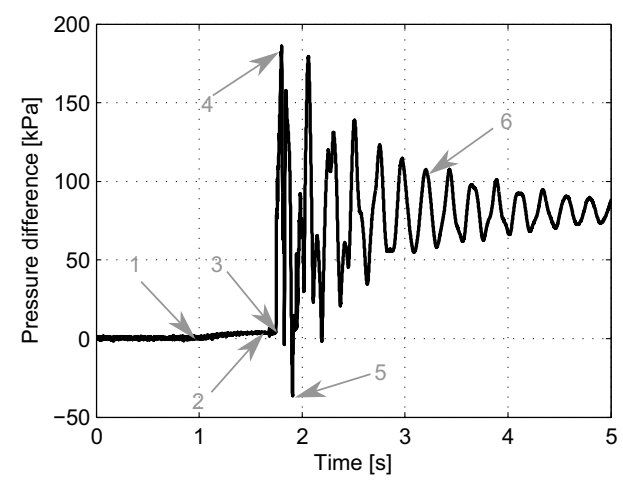

Fig. 9. Pressure difference on the check valve during the transient

The flow rate was evaluated from the pressure records $p_{4}$ and $p_{6}$ by the time-pressure method:

$$
Q(t+\Delta t)=\frac{\Delta t S}{L \rho}\left[p_{4}-p_{5}-R Q^{2}(t)\right]+Q(t),
$$

where the resistance $R$ is not a function of the time, but depends on the initial flow rate (2), which is subject to the numerical optimization.

$$
R=\frac{p_{4}(0)-p_{5}(0)}{Q^{2}(0)} .
$$

The dependence of the velocity on the time (figure 10) is computed from (1) by an appropriate numerical method, when the flow rate at the last time instant equals zero. The solution also includes the initial flow rate and resistance. Then, the start of deceleration (point 1) can be easily found as well as the time, when the flow changed its orientation (point 2). The maximal backward velocity (point 4) is the last information obtained from this graph.

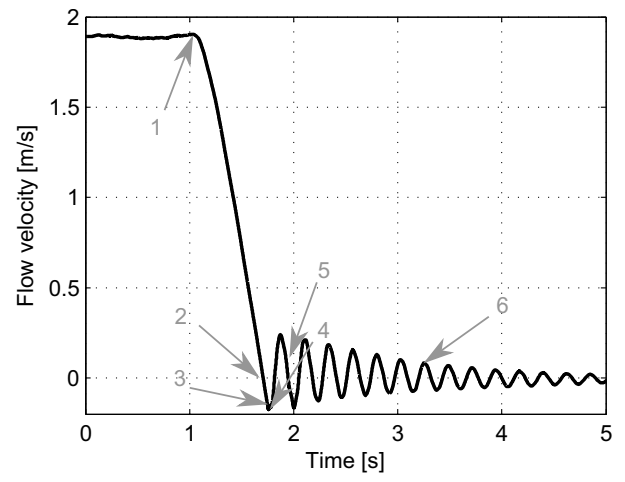

Fig. 10. Flow velocity during the transient

The measurement of the acceleration on the pump body also gives some information about events in the pipeline (see figure 11). The pump disconnection is not visible here (there is not any change of the signal at point 1), because all moving parts kept their moving. The first change is obvious when the speed decreases about $50 \%$. The acceleration amplitude becomes noticeably lower $0.3 \mathrm{~s}$ after the pump disconnection.

Closure of the check valve disc is well visible (point 4) as well as its second closure after reopening (point 5). The pump shaft stop (point 6) is better recognizable than pump disconnection.

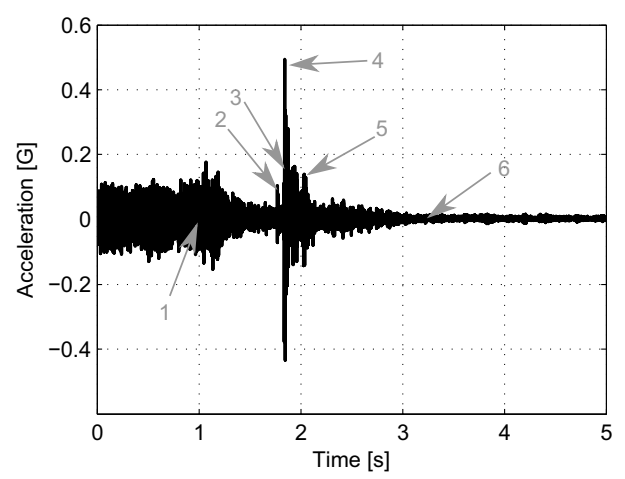

Fig. 11. Measurement of the acceleration on the pump body

The signal from the accelerometer on the check valve body (figure 12) contains strong noise so even the identification of the exact time of disc closure (point 4 ) is difficult. There is a question whether the noise came from mechanical reasons (e. g. pipeline vibrations) or from signal interference. The signal interference is the most probable, because mechanical vibrations would be visible also on the acceleration signal from pump body.

It is interesting that the strongest peak corresponds to the second pressure peak downstream of the check valve (see figure 8), but, at the same moment, the pressure upstream of the check valve is very low. It is the time, when the disc closes for the second time. 


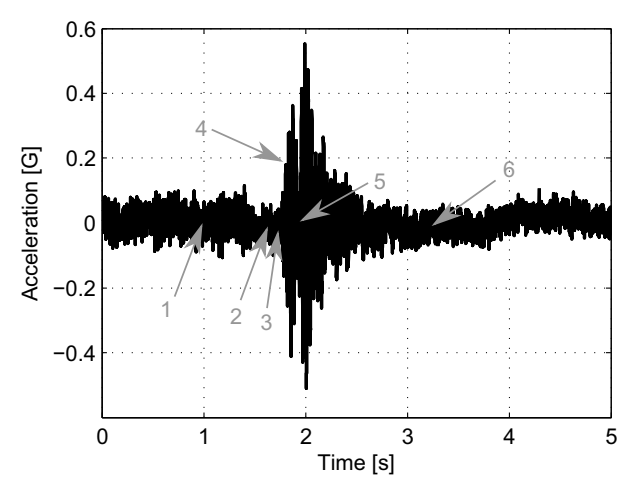

Fig. 12. Measurement of the acceleration on the check valve body

Table 2. Computed deceleration and the maximal reverse velocity

\begin{tabular}{lll}
\hline $\begin{array}{l}\text { Initial flow velocity } \\
\left(\mathrm{m} \mathrm{s}^{-1}\right)\end{array}$ & $\begin{array}{l}\text { Deceleration } \\
\left(\mathrm{m} \mathrm{s}^{-2}\right)\end{array}$ & $\begin{array}{l}\text { Reverse velocity } \\
\left(\mathrm{m} \mathrm{s}^{-1}\right)\end{array}$ \\
\hline 1.3 & 2.4 & 0.15 \\
1.9 & 2.9 & 0.22 \\
2.5 & 3.5 & 0.26 \\
3.4 & 4.1 & 0.34 \\
3.8 & 4.7 & 0.43 \\
\hline
\end{tabular}

The system deceleration can be easily computed from the figure 10 using points 1 and 2. Maximal reverse flow velocity can be read at the same figure. These data are listed in the table 2 for different initial flow velocities.

Figure 13 shows measured data compared with data provided by [9]. The obtained characteristics is found between characteristics of swing check valve and swing flex check valve.

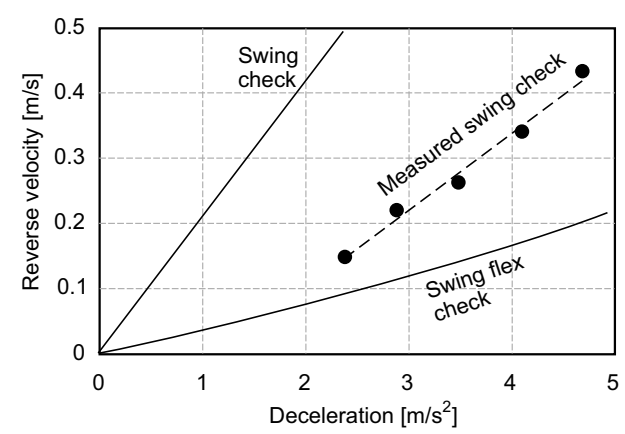

Fig. 13. Maximal reverse velocity dependence on the system deceleration

\section{Discussion}

Following list summarizes important events in the system (the numbering corresponds to the numbers in the figures):

1. Pump disconnection - the pump starts decelerating, which visible on the direct measurement of pump revolutions and pressure, because the pressure change is proportional to the second power of speed change. This point is also well recognizable on the computed flow velocity, but acceleration on the pump body does not give any clue.
2. Change of the flow orientation - can be identified from the computed flow velocity. A low peak can be found also in the signal from the pressure transducer upstream of the check valve.

3. Start of the disc closure - is easily visible on the pressure record at both sides (up- and downstream) of the check valve.

4. End of the disc closure - is easily visible on the pressure record and also on the computed flow rate. It corresponds to the maximal reverse flow velocity. This point could be also identified on the acceleration of the pump body, but, surprisingly, it was very difficult to find exact point also on the acceleration of the check valve body. This record is very noisy.

5. Disc reopening and closing - can be found on the pressure signals and it is visible also on the acceleration of the pump body.

6. Pump shaft stop - is definitely recognizable with scanning the shaft, but its speed before the stop is questionable, because only one mark on the shaft has been used. It is also possible to identify this point on the signal from the accelerometer on the pump body and on the pressure at downstream side of the check valve.

Results show that the higher initial flow the more intense is deceleration after pump disconnection. It can be caused by higher friction loss. It is also apparent that the higher deceleration the higher reverse velocity develops before the check valve closing, because the disc shuts with longer delay. The check valve might not be fully open for lower flow rates, so closes faster. Another explanation could be that higher deceleration causes swirls in the check valve space. These swirls might support the disc and cause delayed closing.

\section{Conclusion}

The paper is focused on the measurement of dynamic characteristic of the swing check valve. An experimental circuit has been built and the swing check valve behaviour was observed during transient events occurring after pump disconnection from the electric network.

Investigated check valve is first of a row of check valves with different constructions, which are going to be tested. This test was supposed to show whether we are able to measure dynamic characteristic of the check valve and if it is possible to describe transient event occurring in the system. The following experiments are going to include also recording of the check valve disc motion.

\section{Acknowledgement}

This work has been supported by Technology Agency of the Czech Republic under the project Innovative research of check valves for extreme operating conditions in energetics TH01011352.

\section{Nomenclature}

$L \quad(\mathrm{~m})$

pipe length between pressure transducers, 


$\begin{array}{lll}p & (\mathrm{~Pa}) & \text { pressure, see figure 5 } \\ Q & \left(\mathrm{~m}^{3} \mathrm{~s}^{-1}\right) & \text { flow rate, } \\ R & \left(\mathrm{~Pa} \mathrm{~s}^{2} \mathrm{~m}^{-6}\right) & \text { resistance } \\ S & \left(\mathrm{~m}^{2}\right) & \text { pipe cross-section, } \\ t & (\mathrm{~s}) & \text { time, } \\ \Delta t & (\mathrm{~s}) & \text { time step, } \\ \rho & \left(\mathrm{kg} \mathrm{m}^{-3}\right) & \text { density. }\end{array}$

\section{References}

1. B. W. Karney, A. R. Simpson, Journal of Hydraulic Research 45547 - 554 (2007)

2. C. Chiu, American Nuclear Society 54, 289 - 291 (1987)

3. J. R. Travis, M. D. Torrey, American Society of Mechanical Engineers winter annual meeting, 1 - 9 (Miami 1985)

4. D. Himr, V. Habán, P. Dokoupil EPJ Web of Conferences 114 (2016) 02038

5. D. Himr, V. Habán, Applied Mechanics and Materials 630, 375 - 382 (2014)

6. K. L. McElhaney, Nuclear Engineering and Design 197, $169-182(2000)$

7. J. V. Ballun Journal of AWWA 99, 3 (2007)

8. W. Rahmeyer Nuclear Industry Check Valve Group, 1996 Winter Meeting, 1 - 10 (St. Petersburg1996)

9. Val-Matic Valve and Manufacturing Corp. Dynamic Characteristics of Check Valves (2003)

10. J. Jablonská, M. Kozubková, EPJ Web of Conferences 114 (2016) 02049

11. M. Vašina, L. Hružík, A. Bureček, EPJ Web of Conferences 114 (2015) 02131 